\title{
Dissimilarity-Based Classifications in Eigenspaces ${ }^{\star}$
}

\author{
Sang-Woon $\mathrm{Kim}^{1}$ and Robert P.W. Duin ${ }^{2}$ \\ 1 Dept. of Computer Science and Engineering, Myongji University, \\ Yongin, 449-728 South Korea \\ kimswamju.ac.kr \\ 2 Faculty of Electrical Engineering, Mathematics and Computer Science, \\ Delft University of Technology, The Netherlands \\ r.p.w.duin@tudelft.nl
}

\begin{abstract}
This paper presents an empirical evaluation on a dissimilarity measure strategy by which dissimilarity-based classifications (DBCs) [10] can be efficiently implemented. In DBCs, classifiers are not based on the feature measurements of individual objects, but rather on a suitable dissimilarity measure among the objects. In image classification tasks, however, one of the most intractable problems to measure the dissimilarity is the distortion and lack of information caused by the differences in illumination and directions and outlier data. To overcome this problem, in this paper, we study a new way of performing DBCs in eigenspaces spanned, one for each class, by the subset of principal eigenvectors, extracted from the training data set through a principal component analysis. Our experimental results, obtained with well-known benchmark databases, demonstrate that when the dimensionality of the eigenspaces has been appropriately chosen, the DBCs can be improved in terms of classification accuracies.
\end{abstract}

\section{Introduction}

Dissimilarity-based classifications (DBCs) [10] are a way of defining classifiers among the classes. The process is not based on the feature measurements of individual objects, but rather on a suitable dissimilarity measure among the objects. The problem with this strategy is that we need to measure the inter-pattern dissimilarities for all the training data to ensure there is no zero distance between objects of different classes. Thus, the classification performance of DBCs relies heavily on how well the dissimilarity matrix is constructed. To improve the performance, therefore, we need to ensure that the dissimilarity matrix is well designed.

With regard to solving this problem, investigations have focused on measuring the appropriate dissimilarity by using various $l_{p}$ norms and traditional measures, such as those used in template matching and correlation-based analysis [10]. In image classification tasks, however, one of the most intractable problems that we encountered when employing these measuring systems is the distortion and lack of information caused by the environmental differences in computation. In face recognition, for example, there

\footnotetext{
* We acknowledge financial support from the FET programme within the EU FP7, under the SIMBAD project (contract 213250). This work was generously supported by the National Research Foundation of Korea funded by the Korean Government (NRF-2011-0002517).
} 
are many kinds of variations based on such factors as pose (direction), expression, illumination, and distance [1], [4].

To address this problem, several strategies, including a generalization of dissimilarity representations [2], [9], a dynamic programming technique [5], a statistical similarity measuring method [6], and classification of regions of interest (ROIs) [13], have been developed and evaluated in the literature. On the other hand, subspace methods of pattern recognition are a technique in which the object classes are not primarily defined as bounded regions in a feature space, but rather given in terms of linear subspaces defined by the principal component analysis (PCA), one for each class [8].

For example, in Eigenface [1], [7], a well-known PCA approach to face recognition, face images are first decomposed into a small set of eigenvectors (i.e., eigenfaces) using a PCA. Then, each individual face is represented in terms of a linear combination of the eigenfaces. Here, the eigenvalues are equal to the variance of the projection of the image data set onto the corresponding eigenvector. Thus, the eigenvectors associated with the higher valued eigenvalues encode the larger variations in the data set, while the eigenvectors associated with the lower valued ones encode smaller variations in the set. Since the latter features encode smaller variations, it is commonly assumed that they represent noise in the data set. From this point of view, when performing DBCs in eigenspaces spanned by the principal eigenvectors, we can expect that the noise could be excluded from the dissimilarity representation.

The major task of our study is to deal with how the dissimilarity measure can be effectively computed. The goal of this paper is to demonstrate that the classification performance of DBCs can be improved by measuring the dissimilarity in the eigenspaces after constructing them by class. In particular, this goal can be achieved by appropriately projecting the data set on the eigenspaces and effectively measuring the distance between the projected points. However, there is an essential difference between what we do in this paper and what Oja [8] (and also O-Alzate, et al. [9]) does. We characterize objects with distances in the subspace, while they use the distances to the subspace.

The remainder of the paper is organized as follows: In Section 2 after providing a brief introduction to DBCs, we present an explanation of the dissimilarity measure used in the eigenspaces and an improved DBC. In Section 3, we present the experimental results obtained with four benchmark image databases and UCI real-life data sets. In Section 4 , we present our concluding remarks.

\section{DBCs in Eigenspaces}

Dissimilarity-based classifications (DBCs): A dissimilarity representation of a set of samples, $T=\left\{\boldsymbol{x}_{i}\right\}_{i=1}^{n} \in \mathbb{R}^{d}$, is based on pairwise comparisons and is expressed, for example, as an $n \times m$ dissimilarity matrix, $D_{T, P}[\cdot, \cdot]$, where $P=\left\{\boldsymbol{p}_{j}\right\}_{j=1}^{m} \in \mathbb{R}^{d}$, a prototype set, is extracted from $T$, and the subscripts of $D$ represent the set of elements on which the dissimilarities are evaluated. Thus, each entry, $D_{T, P}[i, j]$, corresponds to

\footnotetext{
${ }^{1}$ To make it less sensitive to noisy samples, a pseudo-Euclidean embedding method is proposed in [10], where distances are isometrically embedded in a pseudo-Euclidean space and DBCs are performed. The details of the embedding are omitted here, but can be found in [10].
} 
the dissimilarity between the pairs of objects, $\boldsymbol{x}_{i}$ and $\boldsymbol{p}_{j}$, where $\boldsymbol{x}_{i} \in T$ and $\boldsymbol{p}_{j} \in P$. Consequently, an object, $\boldsymbol{x}_{i}$, is represented as a column vector, $\delta\left(\boldsymbol{x}_{i}, P\right)$, as follows:

$$
\delta\left(\boldsymbol{x}_{i}, P\right)=\left[d\left(\boldsymbol{x}_{i}, \boldsymbol{p}_{1}\right), d\left(\boldsymbol{x}_{i}, \boldsymbol{p}_{2}\right), \cdots, d\left(\boldsymbol{x}_{i}, \boldsymbol{p}_{m}\right)\right]^{T}, 1 \leq i \leq n .
$$

Here, the dissimilarity matrix, $D_{T, P}[\cdot, \cdot]$, defines vectors in a dissimilarity space on which the $d$-dimensional object, $\boldsymbol{x}$, is represented as an $m$-dimensional vector, $\delta(\boldsymbol{x})$.

A conventional algorithm for DBCs is summarized in the following:

1. Select the prototype subset, $P$, from the training set, $T$, by using one of the selection methods described in the literature [10].

2. Using Eq. (1), compute the dissimilarity matrix, $D_{T, P}[\cdot, \cdot]$, in which each dissimilarity is computed on the basis of the measures described in the literature [10].

3 . For a testing sample, $\boldsymbol{z}$, compute a dissimilarity column vector, $\delta(\boldsymbol{z})$, by using the same measure used in Step 2.

4. Achieve the classification by invoking a classifier built in the dissimilarity space and operating it on the dissimilarity vector $\delta(\boldsymbol{z})$.

Here, we can see that the performance of DBCs relies heavily on how well the dissimilarity space, which is determined by the dissimilarity matrix, is constructed. To improve the performance, we need to ensure that the matrix is well designed.

Distance Measures in Eigenspaces [7]: The data set, $T$, can be decomposed into subsets, $T_{i}$, as follows 2: $T=\bigcup_{i=1}^{c} T_{i}, T_{i}=\left\{\boldsymbol{x}_{j}\right\}_{j=1}^{n_{i}} \in \mathbb{R}^{d}$, with $n=\sum_{i=1}^{c} n_{i}$, $T_{i} \cap T_{j}=\phi, \forall i \neq j$. Our goal is to design a DBC in an appropriate eigenspace constructed with this training data set, $T$, and to classify a new sample into an appropriate class. To achieve this, for each $T_{i}$, we first find eigenvectors and eigenvalues, $\boldsymbol{\mu}_{i h}$ and $\lambda_{i h},(h=1, \cdots, d)$, of the covariance matrix, $\Sigma_{i}$, using $\Sigma_{i} \boldsymbol{\mu}_{i h}=\lambda_{i h} \boldsymbol{\mu}_{i h}$, and sort them in decreasing order according to the corresponding eigenvalues, i.e., $\lambda_{i 1} \geq, \cdots, \geq \lambda_{i d}$. Next, these eigenvectors are selected to form the row vectors of a transformation matrix, $A_{i}=\left\{\boldsymbol{\mu}_{i h}\right\}_{h=1}^{q} \in \mathbb{R}^{d}$. We then project the data samples, $\boldsymbol{x}_{j},\left(j=1, \cdots, n_{i}\right)$, into $c q$-dimensional subspaces, called eigenspaces, spanned by the arranged principal eigenvectors, using a transformation formula for each class as follows:

$$
\boldsymbol{y}_{i j}=A_{i}^{T}\left(\boldsymbol{x}_{j}-\boldsymbol{m}_{i}\right), \quad 1 \leq i \leq c,
$$

where $\boldsymbol{y}_{i j}=\left(y_{i j 1}, \cdots, y_{i j q}\right)^{T}$ and $\boldsymbol{m}_{i}=\frac{1}{n_{i}} \sum_{j=1}^{n_{i}} \boldsymbol{x}_{j}$, where $\boldsymbol{x}_{j} \in T_{i}$.

Let $\boldsymbol{y}_{i j} \in T_{i}$ and $\boldsymbol{y}_{k l} \in T_{k}$ be $q$-dimensional feature vectors defined in the eigenspace. Many measures exist for $\boldsymbol{y}_{i j}$ and $\boldsymbol{y}_{k l}$, mostly constructed in an additive way after counting the differences for each feature separately. The basic measures come from the family of $l_{p}(p \geq 1)$ distance, $\left\|\boldsymbol{y}_{i j}-\boldsymbol{y}_{k l}\right\|_{p}=\left(\sum_{h=1}^{q}\left(y_{i j h}-y_{k l h}\right)^{p}\right)^{1 / p}$, called Minkowski distance. Based on this distance, various measures can be defined as follows:

1. Manhattan distance $\left(l_{1}\right.$ metrics): $d_{\text {Manh }}\left(\boldsymbol{y}_{i j}, \boldsymbol{y}_{k l}\right)=\sum_{h=1}^{q}\left|y_{i j h}-y_{k l h}\right|$,

2. Euclidean distance $\left(l_{2}\right.$ metrics): $d_{\text {Eucli }}\left(\boldsymbol{y}_{i j}, \boldsymbol{y}_{k l}\right)=\sqrt{\sum_{h=1}^{q}\left(y_{i j h}-y_{k l h}\right)^{2}}$,

3. Sum square error (SSE) distance: $d_{S S E}\left(\boldsymbol{y}_{i j}, \boldsymbol{y}_{k l}\right)=\sum_{h=1}^{q}\left(y_{i j h}-y_{k l h}\right)^{2}$,

4. Canberra distance: $d_{\text {Canbe }}\left(\boldsymbol{y}_{i j}, \boldsymbol{y}_{k l}\right)=\sum_{h=1}^{q} \frac{\left|y_{i j h}-y_{k l h}\right|}{\left|y_{i j h}\right|+\left|y_{k l h}\right|}$.

${ }^{2}$ The subsets have been chosen as the classes here, but clusters could also be used. 
Here, the dimensionality of the eigenspace, $q$, can be selected based on the criteria, such as a heuristic selection [7], a cumulative proportion [8], and an intra-set distance [3]. A criterion associated with the intra-set distance is defined as follow: For an arbitrary sample, $\boldsymbol{x}_{j} \in T_{i}$, the mean of $d\left(\boldsymbol{x}_{j}, T_{i}-\left\{\boldsymbol{x}_{j}\right\}\right)$ over $T_{i}$ is called the intra-set distance of $T_{i}$ and is denoted by $D^{2}\left(T_{i}\right)=\frac{1}{n_{i}} \sum_{j=1}^{n_{i}} d^{2}\left(\boldsymbol{x}_{j}, T_{i}-\left\{\boldsymbol{x}_{j}\right\}\right)$. By conveniently rearranging the elements in the summation of $D^{2}\left(T_{i}\right)$, the intra-set distance can be expressed in terms of the unbiased variances of components of the given samples like: $D^{2}\left(T_{i}\right)=2 \sum_{k=1}^{d} \sigma_{k}^{2}$, where $\sigma_{k}^{2}=\frac{n_{i}}{n_{i}-1}\left(\overline{x_{k}^{2}}-\left(\overline{x_{k}}\right)^{2}\right)$ for all $\boldsymbol{x}_{j} \in T_{i}$. This is the rationale of the scheme for employing the intra-set distance as a criterion to select the dimensionality of the eigenspace. The details of the other criteria are omitted in the interest of compactness, but can be found in the related literature.

Proposed Dissimilarity-Based Classification: To overcome the limitation caused by the variations in illumination and the outlier data, in this paper, we measure the dissimilarities in a transformed subspace, rather than in the input-feature space. The basic strategy of the technique is to solve the classification problem by first mapping the input-feature space to an eigenspace, and then constructing a dissimilarity matrix with the distance measures in the eigenspace; finally, DBCs are performed on the dissimilarity space to reduce the classification error rates. The proposed approach, which is referred to as an eigenspace DBC (EDBC), is summarized in the following:

1. Select the entire training set $T$ as the prototype subset $P$.

2. After computing $A_{i}$ and $\boldsymbol{m}_{i}$ for each class, $T_{i},(i=1, \cdots, c)$, transform the inputfeature vector, $\boldsymbol{x}_{j},\left(j=1, \cdots, n_{i}\right)$, into the feature vectors, $\boldsymbol{y}_{i j}$, using Eq. (2).

3. Using Eq. (1), compute $D_{T, T}[\cdot, \cdot]$, in which each dissimilarity, $d\left(\boldsymbol{x}_{j}, \boldsymbol{x}_{k}\right)$, is measured with $d\left(\boldsymbol{y}_{i j}, \boldsymbol{y}_{l k}\right)$, where the class of the samples $\left\{\boldsymbol{x}_{k}, \boldsymbol{y}_{i j}, \boldsymbol{y}_{l k}\right\}$ is the same.

4. This step is the same as Step 3 in the conventional DBC.

5. This step is the same as Step 4 in the conventional DBC.

The time complexities of the above algorithm, EDBC, can be analyzed as follows: As in the case of DBC, almost all the processing CPU-time of EDBC is consumed in computing the transformation matrix and the dissimilarity matrices. More specifically, in DBC, Step 2 of computing the $n \times n$ dissimilarity matrix requires $O\left(d n^{2}\right)$ time. On the other hand, the computation of that of EDBC needs $O\left(d^{3}+d n^{2}+c n^{2}\right)$ time in executing Steps 2 and 3 .

\section{Experimental Results}

Experimental Data: The proposed method has been tested and compared with the conventional ones. This was done by performing experiments on well-known benchmark databases, namely, Kimia2 (1024/216/2) [11], Yale (1024/165/15) [4], Nist38 (256/200/2) [14], and CMU-PIE (256/1365/65) [12], and other multivariate data sets cited from UCI Machine Learning Repository 3 . Here, three numbers in brackets represent the numbers of dimensions $d$, samples $n$, and classes $c$, respectively. Also, Kimia2 and Nist38 are of binary images, while Yale and CMU-PIE are of gray scale images.

\footnotetext{
${ }^{3} \mathrm{http} / / / \mathrm{www}$. ics.uci.edu/ mlearn/MLRepository.html
} 

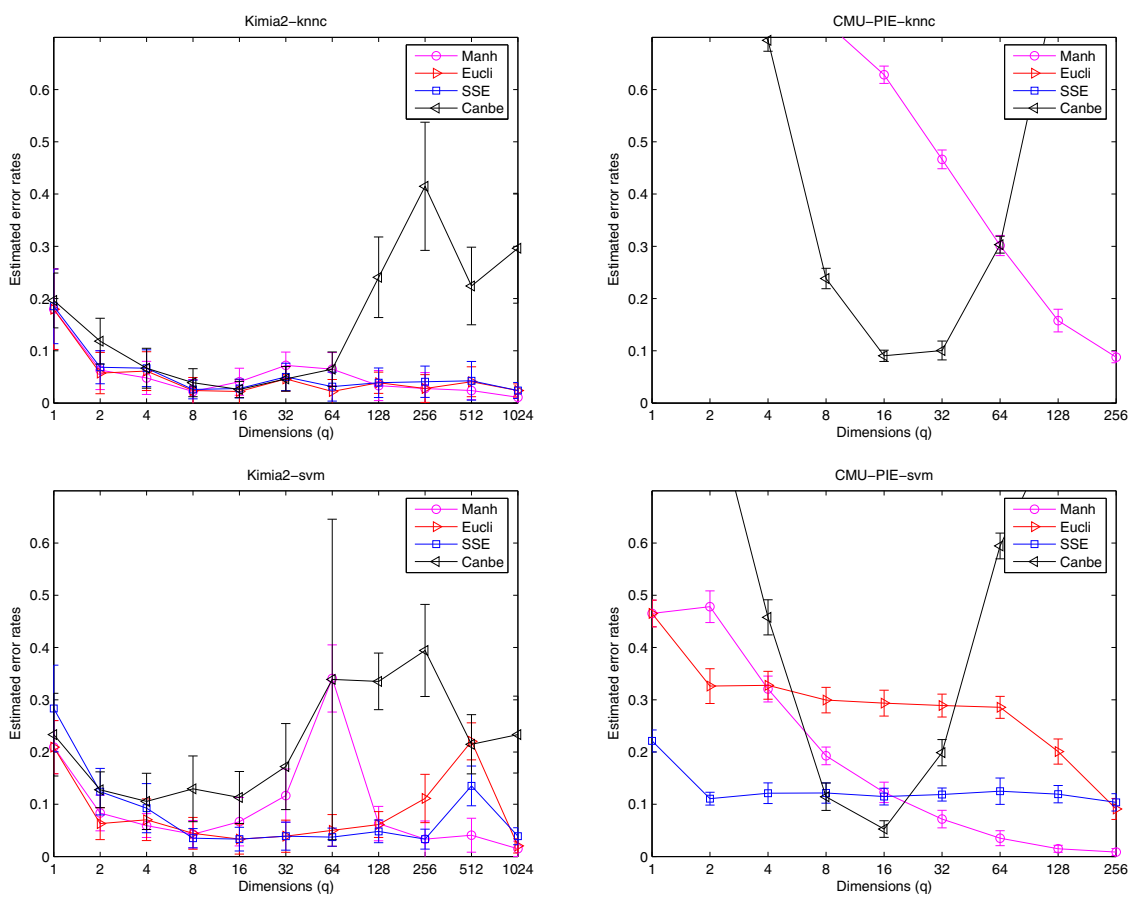

Fig. 1. A comparison of the estimated error rates of knnc and libsvm designed in the eigenspaces: (a) top left, (b) top right, (c) bottom left, and (d) bottom right; (a) and (b) are of knnc, (c) and (d) are of libsvm, obtained with Kimia2 and CMU-PIE using the four distance measures

Experimental Method: In this experiment, first, data sets are randomly split into training sets and test sets in the ratio of $75: 25$. Then, the training and testing procedures are repeated 10 times and the results obtained are averaged. To evaluate the classification accuracies of DBCs designed in the input-feature spaces and the principal eigenspaces, different classifiers, such as $k$-nearest neighbor classifiers and support vector machines, are employed and implemented with PRTools 4 , and will be denoted as knnc and libsvm, respectively, in subsequent sections.

Experimental Results: First, the experimental results obtained in the eigenspaces for Kimia2, Yale, Nist38, and CMU-PIE were probed into. Fig. 1 shows a comparison of the error rates of knnc and libsvm designed in the dissimilarity matrices constructed with the four distance measures in the eigenspaces for the Kimia2 and CMU-PIE databases.

The observations obtained from the figures are the following ones:

- The reader first should observe that the classification accuracy of DBCs can be improved by means of appropriately choosing the distance measures in eigenspaces when the dimensionality of the subspaces is appropriately chosen.

- It should also be pointed out that the error rate of $d_{\text {Canbe }}$, marked with $\triangleleft$ symbol, decreases at first as the dimension increases, but rapidly increases. This overtraining

${ }^{4}$ PRTools is a Matlab toolbox for pattern recognition (refer to http://prtools.org/). 
Table 1. A numerical comparison of the error rates (standard deviations) for the four databases

\begin{tabular}{|c|c|c|c|c|c|}
\hline data sets & distance & \multicolumn{2}{|c|}{$k n n c$} & \multicolumn{2}{|c|}{ libsvm } \\
\cline { 3 - 6 }$(d / n / c)$ & measures & DBCs & EDBCs & DBCs & EDBCs \\
\hline \hline & Manh & $0.0241(0.0232)$ & $0.0111(0.0129)$ & $0.0463(0.0235)$ & $0.0148(0.0146)$ \\
Kimia2 & Eucli & $0.0185(0.0175)$ & $0.0241(0.0125)$ & $0.0204(0.0204)$ & $0.0204(0.0137)$ \\
$(1024 / 216 / 2)$ & SSE & $0.0241(0.0232)$ & $0.0241(0.0152)$ & $0.0463(0.0235)$ & $0.0389(0.0162)$ \\
& Canbe & $0.0241(0.0232)$ & $0.0648(0.0330)$ & $0.0463(0.0235)$ & $0.3389(0.3068)$ \\
\hline & Manh & $0.2267(0.0953)$ & $0.1500(0.0393)$ & $0.2233(0.0668)$ & $0.1500(0.0451)$ \\
Yale & Eucli & $0.2100(0.0903)$ & $0.1800(0.0592)$ & $0.2033(0.0936)$ & $0.1867(0.0450)$ \\
$(1024 / 165 / 15)$ & SSE & $0.2433(0.0969)$ & $0.2067(0.0466)$ & $0.2133(0.0789)$ & $0.1667(0.0351)$ \\
& Canbe & $0.2200(0.0757)$ & $0.2633(0.0618)$ & $0.2200(0.0670)$ & $0.2333(0.0588)$ \\
\hline & Manh & $0.0420(0.0305)$ & $0.0120(0.0103)$ & $0.0520(0.0368)$ & $0.0120(0.0103)$ \\
Nist38 & Eucli & $0.0360(0.0295)$ & $0.0400(0.0249)$ & $0.0380(0.0358)$ & $0.0420(0.0220)$ \\
$(256 / 200 / 2)$ & SSE & $0.0420(0.0305)$ & $0.0540(0.0267)$ & $0.0520(0.0368)$ & $0.0440(0.0280)$ \\
& Canbe & $0.0420(0.0305)$ & $0.1480(0.0634)$ & $0.0520(0.0368)$ & $0.2340(0.0626)$ \\
\hline \multirow{3}{*}{ CMU-PIE } & Manh & $0.7782(0.0148)$ & $0.0877(0.0105)$ & $0.1218(0.0177)$ & $0.0086(0.0066)$ \\
& Eucli & $0.7403(0.0125)$ & $0.7388(0.0096)$ & $0.0923(0.0169)$ & $0.0911(0.0201)$ \\
$(256 / 1365 / 65)$ & SSE & $0.8283(0.0035)$ & $0.8311(0.0169)$ & $0.1114(0.0283)$ & $0.1037(0.0165)$ \\
& Canbe & $0.7055(0.0115)$ & $0.2385(0.0195)$ & $0.1317(0.0188)$ & $0.1145(0.0262)$ \\
\hline
\end{tabular}

observed is the result of the normalization. If many eigenvectors are used, also the ones with small eigenvalues are included. Their contributions are close to the origin and as a result they get large weights: noise is emphasized.

- For the experiment of Kimia2 (and Nist38), which are relatively well represented data sets, the error rates of libsvm show the peaking effect before arriving at the lowest values (refer to Fig. 1 (c)). On the other hand, the error rates of CMU-PIE (and Yale), which are an example of small sample size (SSS) problems, do not show the phenomenon (refer to Figs. 1 (b) and (d)).

- For the experiment of CMU-PIE, in which the face images have been resized into $16 \times 16$ pixels of having only facial components, the error rates obtained with $d_{\text {Canbe }}$ and $d_{M a n h}$ are completely different; the error rates of the latter measure steadily decrease, while those of the former sharply decrease till an optimal or near optimal dimension, but abruptly increase after passing that point (refer to Figs. 1 (b) and (d)). Also, in Fig. 1 b), the error rates of $d_{E u c l i}$ and $d_{S S E}$ do not appear, which means that the two distance measures did not work well for the database. The similar characteristics could also be observed in the Yale and Nist38 databases.

Second, to investigate the difference of DBCs and EDBCs, the experiment (of estimating error rates) was repeated in the two spaces. In this experiment, the dissimilarity matrices were constructed with the four distance measures, where the dimensionality of the matrices was determined as follows: The dimensions of the feature spaces for all DBCs and the $d_{M a n h}, d_{E u c l}$, and $d_{S S E}$ measures of EDBCs are the same as $d$. However, for the $d_{\text {Canbe }}$ measure, it was selected based on the criterion value of the intra-set distance (because of the characteristics shown in Fig. 1). The selected dimensions for Kimia2, Yale, Nist38, and CMU-PIE are 64, 8, 64, and 8, respectively. Table 1 shows a numerical comparison of the error rates of knnc and libsvm for the four databases. 
Table 2. A numerical comparison of the error rates (standard deviations) obtained with $d_{\text {Manh }}$ for UCI data. The results of the other measures are omitted here in the interest of compactness.

\begin{tabular}{|c|c|c|c|c|c|}
\hline data & parameters & \multicolumn{2}{|c|}{$k n n c$} & \multicolumn{2}{|c|}{ libsvm } \\
\cline { 3 - 6 } sets & $(d / n / c)$ & DBCs & EDBCs & DBCs & EDBCs \\
\hline \hline Nist & $(256 / 2000 / 10)$ & $0.0704(0.0086)$ & $\underline{0.0420(0.0098)}$ & $0.0666(0.0092)$ & $0.0428(0.0105)$ \\
Sonar & $(60 / 208 / 2)$ & $0.1980(0.0397)$ & $0.1451(0.0533)$ & $0.1667(0.0455)$ & $0.1588(0.0438)$ \\
Dermatology & $(34 / 366 / 6)$ & $0.0945(0.0260)$ & $0.0714(0.0209)$ & $0.0418(0.0253)$ & $0.0374(0.0221)$ \\
Wine & $(13 / 178 / 3)$ & $0.2372(0.0512)$ & $0.2163(0.0640)$ & $0.0837(0.0745)$ & $0.0744(0.0628)$ \\
Malaysia & $(8 / 291 / 20)$ & $0.4627(0.0480)$ & $0.4610(0.0398)$ & $0.5695(0.0409)$ & $0.5881(0.0582)$ \\
\hline
\end{tabular}

We observed the same characteristics in Table 1 as in Fig. 1 The table clearly shows that the classification accuracies of DBCs can be improved when $d_{\text {Manh }}$ is used to measure the dissimilarity in the eigenspace (see the underlined numbers). However, for $d_{E u c l i}, d_{S S E}$, and $d_{\text {Canbe }}$, the error rates of DBCs and EDBCs are almost the same, which means the increase and/or decrease of the error rates is not significant. Formally, $d_{E u c l i}$ should not change after an eigenvalue decomposition that involves all eigenvectors. The space is just rotated. Then, $d_{E u c l i}$ and $d_{S S E}$ are also rotation independent. However, the values between DBC and EDBC are still slightly different; these might be caused by different training/test set splits in the experiments.

To further investigate the advantage of using the proposed scheme, and, especially, to find out which kinds of significant data set are more suitable for the scheme, we repeated the experiment with a few of UCI data sets. Table 2 shows a numerical comparison of the error rates obtained with $d_{\text {Manh }}$ for the UCI data sets. From the table, it should be observed that the classification accuracy of the proposed scheme can be improved when applied to high-dimensional image data. However, the scheme does not work satisfactorily with low-dimensional data sets.

Additionally, it is interesting to note that PCA was applied separately to each subset $T_{i},(i=1, \cdots, c)$, of the training data, not the entire set. In order to compare two ways of using an eigenspace for each class and a single eigenspace obtained with all training data, we performed two EDBCs for UCI data sets in Table 2. From the experiment, it was observed that the error rates of the latter are generally higher than those of the former. For example, the error rates of the latter knnc and libsvm are 0.0894 and 0.0562 for Nist and 0.2235 and 0.1804 for Sonar, respectively.

In review, it is not easy to crown one particular measuring method with superiority over the others in terms of solving the dissimilarity measuring problem. However, in terms of classification accuracies, the Manhattan distance measured in eigenspaces seems to be more useful for certain kinds of significant data sets than the Euclidean one does. This observation is very interesting. It deserves further discussion and exploration.

\section{Conclusions}

In order to improve the classification performance of DBCs, we studied a distance measuring technique based on eigenspaces of data. To achieve this improvement of DBCs, we first computed eigenvectors and eigenvalues of the training data, one for each class. 
We then performed DBCs in the eigenspaces spanned by the subset of principal eigenvectors, where the dissimilarity was measured with a Manhattan distance. This measuring technique has been employed to solve the distortion and lack of information caused by the differences in illumination and directions. The proposed scheme was tested on four image databases and some UCI data sets. Our experimental results demonstrate that the classification accuracies of DBCs were improved when the dimensionality of the eigenspaces has been appropriately chosen. Although we have shown that the performance of DBCs can be improved by employing the Manhattan distance in eigenspaces, many tasks remain unchallenged. One of them is to further investigate the result that the improvement can be achieved only when using the Manhattan distance. Also, it is not yet clear which kinds of significant data sets are more suitable for the scheme.

\section{References}

1. Belhumeur, P.N., Hespanha, J.P., Kriegman, D.J.: Eigenfaces vs. Fisherfaces: Recognition using class specific linear projection. IEEE Trans. Pattern Anal. and Machine Intell. 19(7), 711-720 (1997)

2. Bicego, M., Murino, V., Figueiredo, M.A.T.: Similarity-based classification of sequences using hidden Markov models. Pattern Recognition 37, 2281-2291 (2004)

3. Friedman, M., Kandel, A.: Introduction to Pattern Recognition - Statistical, Structural, Neural and Fuzzy Logic Approaches. World Scientific, New Jersey (1999)

4. Georghiades, A.S., Belhumeur, P.N., Kriegman, D.J.: From few to many: Illumination cone models for face recognition under variable lighting and pose. IEEE Trans. Pattern Anal. and Machine Intell. 23(6), 643-660 (2001)

5. Kim, S.-W., Gao, J.: A Dynamic Programming Technique for Optimizing DissimilarityBased Classifiers. In: da Vitoria Lobo, N., Kasparis, T., Roli, F., Kwok, J.T., Georgiopoulos, M., Anagnostopoulos, G.C., Loog, M. (eds.) S+SSPR 2008. LNCS, vol. 5342, pp. 654-663. Springer, Heidelberg (2008)

6. Kim, S.-W., Duin, R.P.W.: On improving dissimilarity-based classifications using a statistical similarity measure. In: Bloch, I., Cesar Jr., R.M. (eds.) CIARP 2010. LNCS, vol. 6419, pp. 418-425. Springer, Heidelberg (2010)

7. Moon, H., Phillips, P.J.: Computational and performance aspects of PCA-based facerecognition algorithms. Perception 30, 303-321 (2001)

8. Oja, E.: Subspace Methods of Pattern Recognition. Research Studies Press (1983)

9. Orozco-Alzate, M., Duin, R.P.W., Castellanos-Dominguez, G.: A generalization of dissimilarity representations using feature lines and feature planes. Pattern Recognition Letters 30, 242-254 (2009)

10. Pekalska, E., Duin, R.P.W.: The Dissimilarity Representation for Pattern Recognition: Foundations and Applications. World Scientific Publishing, Singapore (2005)

11. Sebastian, T.B., Klein, P.N., Kimia, B.B.: Recognition of shapes by editing shock graphs. In: Proc. of the 8th IEEE Int'l Conf. on Computer Vision, Vancouver, Canada, pp. 755-762 (2001)

12. Sim, T., Baker, S., Bsat, M.: The CMU pose, illumination, and expression (PIE) database of human faces, Technical Report RI-TR-01-02, Carnegie Mellon Univ., Pittsburgh, PA (2001)

13. Sørensen, L., Loog, M., Lo, P., Ashraf, H., Dirksen, A., Duin, R.P.W., de Bruijne, M.: Image dissimilarity-based quantification of lung disease from CT. In: Jiang, T., Navab, N., Pluim, J.P.W., Viergever, M.A. (eds.) MICCAI 2010. LNCS, vol. 6361, pp. 37-44. Springer, Heidelberg (2010)

14. Wilson, C.L., Garris, M.D.: Handprinted Character Database 3. Technical Report, National Institute of Standards and Technology, Gaithersburg, Maryland (1992) 